International Journal of Modern Physics B, Vol. 10, No. 5 (1996) 599

(c) World Scientific Publishing Company

\title{
ERRATA
}

\section{FERROELECTRIC LIQUID CRYSTALS: FROM THE PLANE WAVE TO THE MULTISOLITON LIMIT}

[INT. J. MOD. PHYS. B, Vol. 9, Nos. 18 \& 19 (1995) 2321 2362]

I. MUŠEVIČ, B. ŽEKŠ, R. BLINC and TH. RASING

Figure 9 should be replaced by

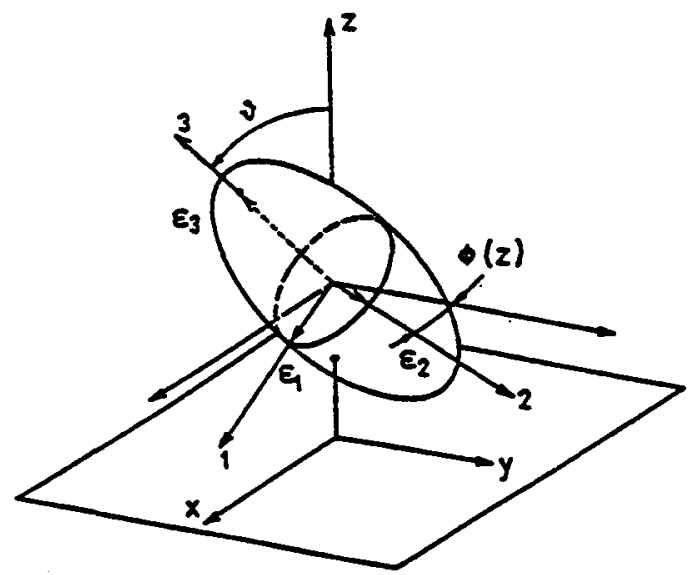

Fig. 9. Local orientation of the dielectric tensor in the $\mathrm{SmC}^{*}$ phase.

The sentence "By symmetry arguments...." at p. 2336 is rewritten as "... one of the axis of the eigenframe $(1,2,3)$ (e.g. $\left.2 \| C_{2}\right)$ must be parallel ...."

Equation (31) should read as follows:

$$
|\mathbf{k}, p\rangle=|\mathbf{k}, p\rangle_{0}+\sum_{\mathbf{k}^{\prime}, p^{\prime}} \frac{\omega_{0}^{2}(\mathbf{k}, p)}{\omega_{0}^{2}\left(\mathbf{k}^{\prime}, p^{\prime}\right)-\omega_{0}^{2}(\mathbf{k}, p)} \frac{\left\langle\mathbf{k}^{\prime}, p^{\prime}\left|\varepsilon_{0}^{-1} \delta \varepsilon\right| \mathbf{k}, p\right\rangle_{0}}{\left\langle\mathbf{k}^{\prime}, p^{\prime} \mid \mathbf{k}^{\prime}, p^{\prime}\right\rangle_{0}}\left|\mathbf{k}^{\prime}, p^{\prime}\right\rangle_{0}
$$

Equation (45) should read as follows:

$$
\tau_{A}^{-1}=\frac{2 \alpha}{\gamma}\left(T_{\mathrm{c}}-T\right)+\frac{K_{3}}{\gamma}\left(q_{\mathrm{c}} \pm q\right)^{2}
$$

